KARL JANSEN-WINKELN

\title{
Ein Priester als Restaurator
}

\author{
Zu einer ptolemäischen Inschrift am Luxortempel
}

\author{
Hierzu Tafel XV-XVI
}

Auf dem nordöstlichen Teil ${ }^{1}$ der Außenwand des Vorhofes Amenophis' III. im Luxortempel befindet sich ein „Graffito" eines Amunpriesters namens K3p.f-h3-Mntw ${ }^{2}$, mit „schönem Namen“" P3-dj-Jmn-Jpt, Sohn eines Ns-Mnw und einer St 3 jrt-bjnt, in der er erwähnt, daß er ein Prozessionsbild und einen Schrein für Amenope neu anfertigen ließ. Diese Inschrift ${ }^{3}$ ist von G. Daressy veröffentlicht worden ${ }^{4}$, allerdings mit einer ganzen Reihe von Fehlern und Ungenauigkeiten.

Rechts neben der bekannten Bauinschrift des 'nh-p3-hrd aus dem Jahr 4 des Philipp Arrhidäus, s. PM $\mathrm{II}^{2}, 335$ (219b) sowie M. Abder-Raziq, ASAE 69, 1983, $211-8$.

H. de Meulenaere, Le surnom égyptien à la Basse époque, Istanbul 1966, 21 (66); 20, n. 96 erwägt die Alternativen K3p.f-h3-Jmn und K3p.f-h3-Mntw. Der Name kommt in dem "Graffito" viermal vor: 1 . oben rechts, in der Rahmenzeile, 2. in Z. 2 der längeren Inschrift rechts, 3. über dem knienden Beter, 4. in Z. 7 der kürzeren Inschrift links. In Beleg 2 und 4 hängt hinten an der Federkrone etwas herab, das man als das $\mathrm{B}$ and an der Krone des Amun deuten könnte. In Beleg 1 hat der Gott aber wohl einen Falkenkopf, vor allem aber deutlich zwei Uräen, und auch in Beleg 3 und 4 werden zwei Uräen gemeint sein. Daher ist wohl Mntw $z u$ lesen (s. LÄ IV, 200 und v. a. J. Leclant, Mél. Masp., I, 4, 1961, 75-9), und das hinten herabhängende "Band" dürfte dann der Schwanz der Stirnschlangen Sein. Zu den Namen des Typs K3p.f-h3+ Gottesname vgl. allgemein auch M. Thirion, RdE 42, 1992, 231.

Die Bezeichnung „Graffiti“ für die Texte auf dieser Mauer in $\mathrm{PM} \mathrm{II}^{2}, 335$ bezieht sich zum einen darauf, daß sie nicht zur ,regulären“ Dekoration gehören, zum anderen sind einige davon tatsächlich sehr unregelmäBig ausgeführt. Die hier besprochene Inschrift zeigt eine sorgfältige und wohlüberlegte Disposition, so daß man kaum von einem Graffito sprechen kann.

RecTrav 14, 1893, 34 (LV). Vgl. auch M. Dores se, RdE 25, 1973, 129; 134.
Eine Kollation Sethes für das Wörterbuch ${ }^{5}$ enthält wesentliche Verbesserungen, aber gerade an einigen für das Verständnis besonders wesentlichen Stellen ist auch Sethes Abschrift nicht in Ordnung. Da zudem nie eine brauchbare Übersetzung des Textes erschienen ist ${ }^{6}$, bedarf eine neue Bearbeitung wohl keiner Rechtfertigung. Zur Datierung des Graffitos läßt sich nur sagen, daß es mit größter Wahrscheinlichkeit in die Ptolemäerzeit gehört?

\section{Übersetzung}

Rahmenzeile, rechts und oben, in größeren Hieroglyphen:

Der Gottesvater des Amun in Karnak(1) $K 3 p . f-h 3-M n t w(2)$, sein schöner Name ist P3-djJmn-Jpt, der Sohn des Gottesvaters und Reinigers $N s-M n w(3)$, geboren von der Musikerin des Amun-Re St 3 -jrt-bjnt(4), indem er sagt:

\section{Umrandetes Rechteck vor dem Beter:}

1 O Amenope, Stier mit erhobenem Arm, männlichster der Götter, großer lebender Gott, Oberster der Götter(5), jenes Abbild des $\operatorname{Re}(6)$, richte dein schönes Gesicht 2 auf deinen Diener!

${ }^{5}$ Wb-Mappe 243, Zettel 722-729.

Der Übersetzungsversuch von M. Abder-Raziq, ASAE 69, 1983, 215-6 basiert auf dem unvollständigen und fehlerhaften Text von Daressy.

So auch De Meulenaere, Le surnom égyptien, 21(66); 24. 
Mögest du angesehen sein lassen(7) den Gottesvater und Propheten des Amun in Karnak K3p.f$h 3-M n t w(2)$, sein schöner Name ist P3-dj-Jmn-Jpt, den Sohn des Gottesvaters und Reinigers(8) in deinem Haus $N s-M n w$, geboren von der Musikerin des Amun-Re St 3 -jrt-bjnt, 3 indem er sagt: „Ich bin dein Diener, der tut, was dein Herz liebt, der Lobpreis gibt deinem schönen Gesicht. Ich hörte nicht auf, deinem Haus zu dienen, ohne daß es einen Tadel von seiten deiner Majestät gab(9). Ich tat die Maat, die du liebst, 4 ein Abscheu für mich war das Böse(10), der Abscheu deines $\mathrm{Ka}$. Ich stellte den großen Gott(11), den Ersten seines Harims, mit seinen Hymnen zufrieden, und er ließ mich die Sänfte(?) seiner Überfahrten(?) sehen(12). O WabPriester, 5 die in diesen Tempel eintreten werden: Laßt mich(13) unter den vortrefflichen Begünstigten(14) sein, dann wird euch der große lebende Gott begünstigen, der Oberste der Götter, bei seinen Zeremonien 6 zu Beginn der Dekade(15), und die Namen(16) werden ständig bleiben und dauern im Tempel des Amenope(?) [Kamut]ef, (des Ersten) (seines) Harims(?)(17) in alle Ewigkeit.

\section{Umrandetes Rechteck hinter dem Beter:}

Ich habe dieses Prozessionsbild(18) in Verfall begriffen gefunden. Ich errichtete es (von neu$\mathrm{em})$, entsprechend wie es vordem gewesen war. Ich habe (damit) seinen [des Gottes] ,Leib' verjüngt zu neuem Leben, ich habe seinen $\mathrm{Ka}$ zufriedengestellt durch das, was er liebt. Ich habe ihm einen Schrein(19) gemacht aus $\check{s}$-Holz, um den ,Leib، seines Prozessionsbildes damit zu umfassen(20). Möge er Leben, Heil und Gesundheit geben, eine lange Lebenszeit, ein langes und glückliches Alter sowie Zufriedenheit und Freude tagtäglich für den Gottesvater K3p.f-ḩ3$M n t w$, sein schöner Name ist P3-dj-Jmn-Jpt.

\section{Um den Beter:}

(oben:) Der Gottesvater K3p.f-h3-Mntw w sein schöner Name ist P3-dj-Jmn-Jpt, (links:) der Sohn des $N s-M n w$, geboren von der St 3 -jrt- bj(n)t(21), (rechts:) der tagtäglich tut, was sein Gott Amenope liebt. (unten, Z. 1:) Er hat jeden Baum wachsen lassen [für] Amun(?)(22), (Z. 2) den Herrscher, seinen Herrn, damit Schatten entstehe durch ihn täglich(23).

\section{Anmerkungen}

1) Die Gruppe über dem Rücken des ist mir nicht erklärbar. Der übliche Titel ist einfach jtj-ntr (n) Jmn m Jpt-swt, ohne jeden Zusatz nach Jmn.

2) Zur Lesung des Gottesideogramms s. o., Fußnote 2.

3) Zur Schreibung des Namens s. H. de Meulenaere, BIFAO 54, 1954, 73-82, bes. 78. Man könnte das 9 des vorhergehenden $j t j$ $n t r$ auch doppelt lesen und so den zweiten Titel als ${ }^{b} b w-n t r$ verstehen. Das wäre die üblichere Form, aber ${ }^{c} b w$ kann auch allein vorkommen, vgl. Wb I, 175,11 und K. Jansen-Winkeln, Biographische und religiöse Inschriften der Spätzeit, ÄUAT 45, 2001, 116-7 (2).

4) Das $\gamma$ ist offenbar aus den demotischen Schreibungen des Namens übernommen, s. Spiegelberg, ZÄS 59, 1924, 150-4; E. Lüddeckens (ed.), Demotisches Namenbuch, I, 13, Wiesbaden 1995, 943-4.

5) Vgl. zu dieser Bezeichnung Sethe, Amun, $\int 111$.

6) Man ist zunächst versucht, den Gebrauch des Demonstrativs $p w$ durch den Vokativ bedingt zu sehen, vgl. H. Grapow, Wie die Alten Ägypter sich anredeten, I, APAW 1939, 18-21. Das kann aber nicht richtig sein, denn auf einer Statue in Linköping heißt Amenope in einer Opferformel (also kein Vokativ) znn pw n Hr-z3-3st ,dieses Abbild des Harsiese c8 $^{8}$, ebenso auf dem Würfelhocker Kairo JE 38013 (BASK 9, 2003, 4). Bemerkenswert ist die seltene Kombination von "neuem“ und , altem“ Demonstrativpronomen ( $p 3$ und $p w$ ), s. dazu Grapow, op. cit., 21.

${ }^{8}$ G. Björkman, The Smith Collection of Egyptian Antiquities, Uppsala 1971, 13, Fig. 2, Z. 1. 
7) S. Wb I, 262,7.

8) Vgl. oben, Anm. 3. Das $n$ gehört wohl hinter $z$.

9) Oder „für, an deinen Diener“? Allerdings wird $h m$,Diener" in dieser Inschrift sonst geschrieben.

10) Wohl eher $j z\langle f\rangle(t)$ zu lesen (vgl. auch Wb I, 129,2 und P. Wilson, A Ptolemaic Lexicon, OAL 78, 1997, 111) als jzw ,die Bö-

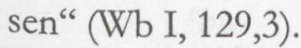

11) Der abrupte Übergang von der 2. in die 3. Person ist merkwürdig.

12) Die beiden Gruppen nach $m 33 . j$ sind ziemlich undeutlich geschnitten, v. a. das als $\downarrow$ interpretierte Zeichen ist sehr unsicher. Die Lesung des mit „Sänfte“ übersetzten Wortes bzw. Ideogramms (Falke auf Bahre?) ist unsicher; das Determinativ von sšmw (s. u.) sieht ganz anders aus. Immerhin könnte der in einem Tragegestell transportierte Schrein des ,verhüllten“ Amenope gemeint sein, vgl. dazu M. Dores se, RdE 23, 1971, 113136; 25, 1973, 92-135. In jedem Fall dürfte das, was $K 3 p . f-h 3-M n t w$ als "Gegenleistung“ für seine Gottesverehrung sehen durfte, ein Utensil dieses Gottes sein, das als besonders heilig und ,geheim" galt.

13) Zur Schreibung von wj vgl. JansenWinkeln, Biographische und religiöse Inschriften, 154 (41).

14) Die Art des Personendeterminativs hinter 8 إ ist nicht deutlich. Mit $h z j w$ werden oft diejenigen Toten bezeichnet, die eine Statue im Tempel aufstellen durften. Eine entsprechende Bitte wird im übrigen sonst an den Gott gerichtet, nicht an die Priester.

15) D. h. die Überfahrt des Amenope zum kleinen Tempel von Medinet $\mathrm{Habu}$ und die damit verbundenen Riten, vgl. Doresse, RdE 25, 1973, 121-135; 31, 1979, 36-65; C. Traunecker u. a., La chapelle d'Akôris à Karnak, II, Paris 1981, 130-4.

16) Man sollte ein Suffix nach $r n(w)$ erwarten, entweder $r n . t n$, „eure Namen" oder, falls der Sprecher sich selbst einbezieht, $r n . n$, unsere Namen“. Vielleicht ist die Stelle tatsächlich in $\frac{9}{11} \mathrm{zu}$ emendieren.

17) $\mathrm{Ob} z u \stackrel{D_{u}}{\square} \square \square$ zu ergänzen ist? Über dem Kopf der Schlange sind noch Reste sichtbar, bei denen es sich um die Beine eines Vogels handeln könnte. Falls man so ergänzt, müßte das $f$ sowohl bei $K 3-m w t . f$ als auch bei hntj jpt.f zu lesen sein, ähnlich wie auf der Statue Kairo JE 37993 (s. Jansen-Winkeln, Biographische und religiöse Inschriften, 374; 105 [2]). Der vorhergehende Gottesname ist entweder $4 \overline{w_{0}}$ oder

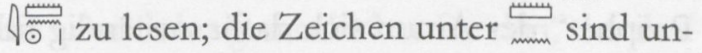
deutlich geschnitten.

18) Zu sšmw als in einer Kultbarke mitgeführtem und in einem Schrein bewahrtem „Prozessionsbild“"vgl. B. Ockinga, Die Gottesebenbildlichkeit im Alten Ägypten und im Alten Testament, ÄUAT 7, 1984, 41-5. Zum Aussehen dieses Kultbildes des Amenope (die Hieroglyphe hier und die in Z. 5 lassen die Details nicht genau erkennen) s. M. Doresse, RdE 23, 1971, 113-136; 25, 1973, 92-135.

19) Es ist natürlich zu $k 3 r$ (Ц山) $\mathrm{zu}$ emendieren.

20) : könnte sowohl jwf als auch $h^{\complement} w$ gelesen werden, vgl. Wb I, 51,15-6; III, 38,16. Gemeint ist auf jeden Fall der Schrein, der die in der Barke mitgeführte Statue verbirgt, vgl. Doresse (s. vorletzte Anm.).

21) Zur Schreibung von bjnt ohne $n$ vgl. K. Jansen-Winkeln, Spätmittelägyptische Grammatik, ÄUAT 34, 1996, §54.

22) Die Reste am Ende der Zeile sind nicht sicher zu identifizieren, es könnte eine (kursive?) Schreibung von ${ }_{m}$ sein. Auf jeden Fall muss hier eine Gottesbezeichnung gestanden haben, auf die sich das folgende $j t j j$ $n b . f$ beziehen kann.

23) Zum Verweis auf $s 3 n b$ mit einem singularischen Suffix s. Gardiner, EG, $₫ 510,2$. Das $\subset$ ist hier seitenverkehrt geschrieben, ebenso wie in dem umrandeten Rechteck hinter dem Beter in Z. $2\left(\underline{d}^{\top} m\right)$ und 3 ( $m$ whm ${ }^{\prime} n h$ ). Sinn des ganzen Abschnittes ist es, daß K3p.f-ḩ-Hnzw Bäume gepflanzt hat, die Schatten spenden sollen. Das dürfte am ehesten entlang der Prozessionsstraße des Amenope sinnvoll gewesen sein, damit die Priester seine Barke nicht in der prallen Sonne zu schleppen hatten. 
Es ist erstaunlich, daß sich in diesem Text ein einfacher Priester rühmt, eine Art Allee beim Tempel angelegt und Schrein und Kultbild erneuert zu haben. Das waren eigentlich königliche Aufgaben. Es sagt einiges über die ärmlichen Verhältnisse der thebanischen Provinz in der (wohl schon fortgeschrittenen) Ptolemäerzeit aus, daß ein schlichter „Gottesvater und Prophet" des Amun sich dafür zuständig fühlen konnte. Zweifellos deshalb durfte er auch an dieser Stelle eine längere Inschrift anbringen; eine Stele im Tempel wäre wohl wiederum zu aufwendig gewesen.

Im Museum Linköping gibt es einen Würfelhocker der Ptolemäerzeit, der einem Ns-Mnw gehört, dem Sohn eines K3p.f-ḩ3-Jmn (nach bisheriger Lesung, s. u.) und einer $N s-H r-p 3-R^{c^{9}}$. Dieser Mann hatte noch mindestens eine weitere Statue, den Würfelhocker Baltimore WAG $22.183^{10}$. In den Inschriften der Statue aus Linköping (nicht aber in denen auf dem Würfelhocker in Baltimore) spielt der Gott Amenope in seinen verschiedenen Ausformungen eine zentrale Rolle. Wohl nicht zuletzt deshalb hat man in dem Vater des Besitzers der beiden Statuen, dem angeblichen K3p.f- $h 3-J m n$, dieselbe Person erkennen wollen, die das "Graffito" in Luxor anbringen lie ${ }^{11}$. Unter der Voraussetzung, daß diese Person tatsächlich K3p.f-h3-Jmn hieß ${ }^{12}$, ergäbe sich also folgender Stammbaum:

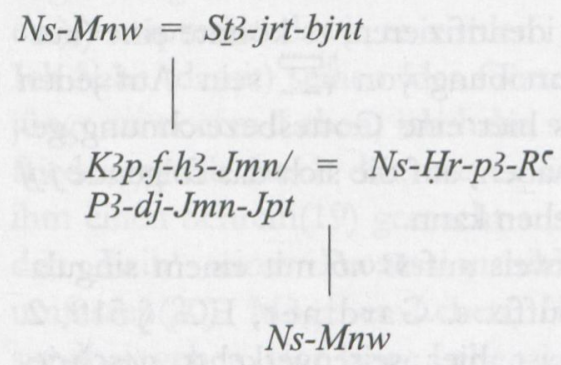

Ns-Mnw

${ }^{9}$ S. G. Björkman, The Smith Collection, 8-22 (102).

G. Steindorff, Catalogue of the Egyptian Sculpture in the Walters Art Gallery, Baltimore 1946, 57-8; pl. XXIX; CXVI (168).

${ }^{11}$ Björkman, The Smith Collection, 8-9; ebenso M. Doresse, RdE 25, 1973, 93.

${ }^{12}$ Was unwahrscheinlich ist, aber vielleicht nicht völlig auszuschließen, s. o., Fußnote 2.
Das wäre möglich, aber keineswegs zwingend (für den Vater des Besitzers der Statuen wird kein Beiname erwähnt). Die umgekehrte Reihenfolge, der Auftraggeber des Graffitos als Sohn des Besitzers der Statuen wäre ebensogut denkbar:

\section{$K 3 p \cdot f-h 3-J m n=N s-H r-p 3-R^{c}$ \\ $N s-M n w=S \underline{t} 3-j r t-b j n t$ \\ K3p.f-h3-Jmn/ P3-dj-Jmn-Jpt}

In beiden Fällen wären, wie so häufig, Großvater und Enkel gleichnamig.

Tatsächlich war es aber wohl ein K3p.f-h3$M n t w$, der das Graffito anbringen lie $\beta^{13}$, und auch der Vater des Besitzers der beiden Statuen heißt kaum K3p.f-h3-Jmn. Auf der Statue in Linköping wird sein Name an folgenden Stellen geschrieben: Vorderseite, oben rechts ${ }^{14}$, rechte Seite, Z. $4^{15}$, linke Seite, Z. $4^{16}$, Rückseite, Z. $1^{17}$. Für die ersten beiden Stellen gibt das Faksimile von Björkman 部, für die beiden letzten der Statue in Baltimore erscheint der Name nur in Z. 8 der Vorderseite. Steindorff ${ }^{18}$ gibt das

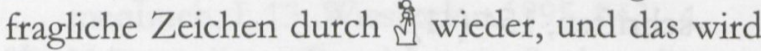
durch ein Foto des Museums bestätigt (obwohl auch nicht auszuschließen ist). Ebenso wird der gleichlautende Name des Enkels in Z. 3 auf der rechten Seite (s. u.) geschrieben. In beiden Fällen ist eine Doppelfederkrone definitiv ausgeschlossen. Die in dem Graffito und auf den Statuen genannten Personen haben also folgende Namen und Titel:

${ }^{13}$ S. o., Fußnote 2.

${ }^{14}$ Björkman, The Smith Collection, 17, Fig. 3.

15 Ibid., 13, Fig. 2 (!).

${ }^{16}$ Ibid., 10, Fig. 1 (!).

${ }^{17}$ Ibid., 20, Fig. 4.

${ }^{18}$ Catalogue of the Egyptian Sculpture, pl. CXVI (168A). 
Graffito:

\begin{tabular}{|c|c|}
\hline $\begin{array}{l}\text { Besitzer: } \\
K 3 p . f-h 3-M n t w \\
r n . f n f r \text { P3-dj- } \\
\text { Jmn-Jpt }\end{array}$ & $\begin{array}{l}j t j-n \underline{t r} \text { hm(-ntr) Jmn m Jpt-swt / } \\
j t j-n \underline{t} r \mathrm{n} \text { Jmn } m \text { Jpt-swt / jtj-ntrr }\end{array}$ \\
\hline $\begin{array}{l}\text { Vater: } \\
N_{s-M n w}\end{array}$ & $\begin{array}{l}j t j-n t r \\
r b w\end{array}$ \\
\hline $\begin{array}{l}\text { Mutter: } \\
\text { St } 3 \text {-jrt-bjnt }\end{array}$ & $j h y t$ Jmn-Re \\
\hline
\end{tabular}

Statuen in Linköping und Baltimore:

\begin{tabular}{|c|c|}
\hline $\begin{array}{l}\text { Besitzer: } \\
N_{s-M n w}\end{array}$ & 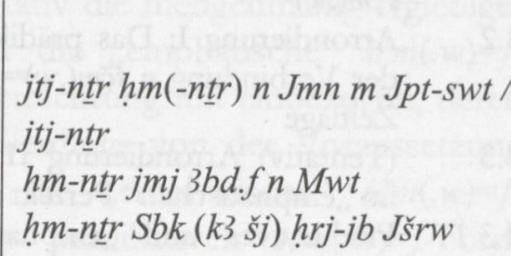 \\
\hline $\begin{array}{l}\text { Vater: } \\
\text { K3p.f-h3-Hnzw }\end{array}$ & 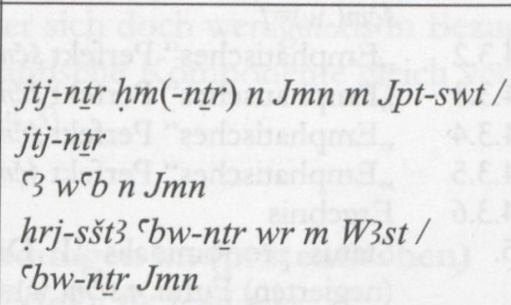 \\
\hline $\begin{array}{l}\text { Mutter: } \\
N_{S-H r}- \\
p 3-R^{r}\end{array}$ & $\begin{array}{l}n b t-p r \\
j h y t J m n-R^{r}\end{array}$ \\
\hline
\end{tabular}

$\mathrm{Zu}$ dem Würfelhocker in Baltimore ist noch zu bemerken, daß auf ihm (außerhalb der Filiationen) auch noch ein Sohn namens K3p.f-h3Hnzw genannt wird. Auf der rechten Seite heißt es in Z. 3-4 ${ }^{19}$ : 4 „(Mein) Sohn, der Gottesvater K3p.f-h3-Hnzw, gerechtfertigt, hat mir meinen ,Leib' ersetzt, derjenige, der meine Statue errichtet hat. Gut ist das, was der Sohn gemacht hat für den, der ihn erzeugte." Es ergibt sich also eine Folge K3p.f$h 3-H n z w-N s-M n w-K 3 p . f-h 3-H n z w^{20}$.

Gemeinsam ist dem Graffito und den Statuen also nur ein "Gottesvater" namens $N s-M n w$, in dieser Zeit einer der häufigsten thebanischen Namen überhaupt. Und wenn sowohl in dem Graffito als auch auf der Statue in Linköping der Gott Amenope eine wichtige Rolle spielt, muß das ja noch nicht bedeuten, daß beide Priester zur selben Familie gehören (obwohl vermutlich die gesamte höhere thebanische Priesterschaft mehr oder weniger verwandt war).

\section{SUMMARY}

Republication of an inscription on the outer wall (east side) of the forecourt of Amenophis III in the temple of Luxor, composed by a priest of Amun in the Ptolemaic period. He boasts of having (re)built the processional image of Amenope and its shrine, and of having planted trees in the temple area to create a shadow.

Steindorff, Catalogue of the Egyptian Sculpture, pl. 116 (168 C), korrigiert nach Fotos des Museums.

${ }^{20}$ Im demotischen Papyrus Philadelphia 6, vso 6,9 aus dem 18. Jahr Ptolemaios' I. (287) kommt auch ein $K 3 p . f-h 3-H n z w$, Sohn eines $N s-M n w$ vor (Hinweis G. Vittmann), s. M. El-Amir, A Family Archive from Thebes, Kairo 1959, 34 sowie E. Lüddeckens (ed.), Demotisches Namenbuch, I, 13, Wiesbaden 1995, 1006, aber beide Namen sind recht häufig. 


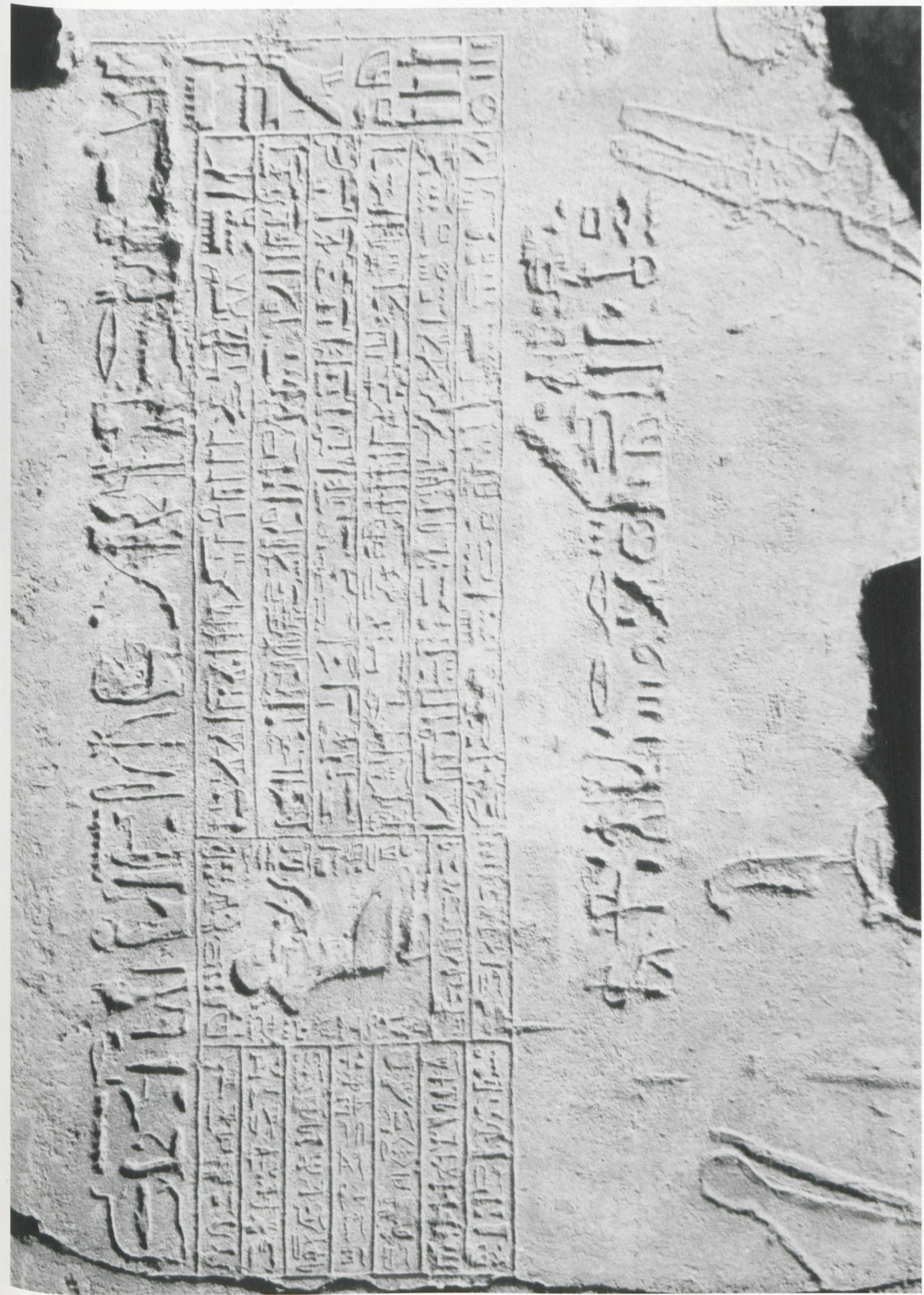

Inschrift des Amunpriesters K3p.f-h3-Mntw, Luxortempel, Vorhof Amenophis' III., Foto des Verfassers (zu Jansen-Winkeln, Ein Priester als Restaurator) 


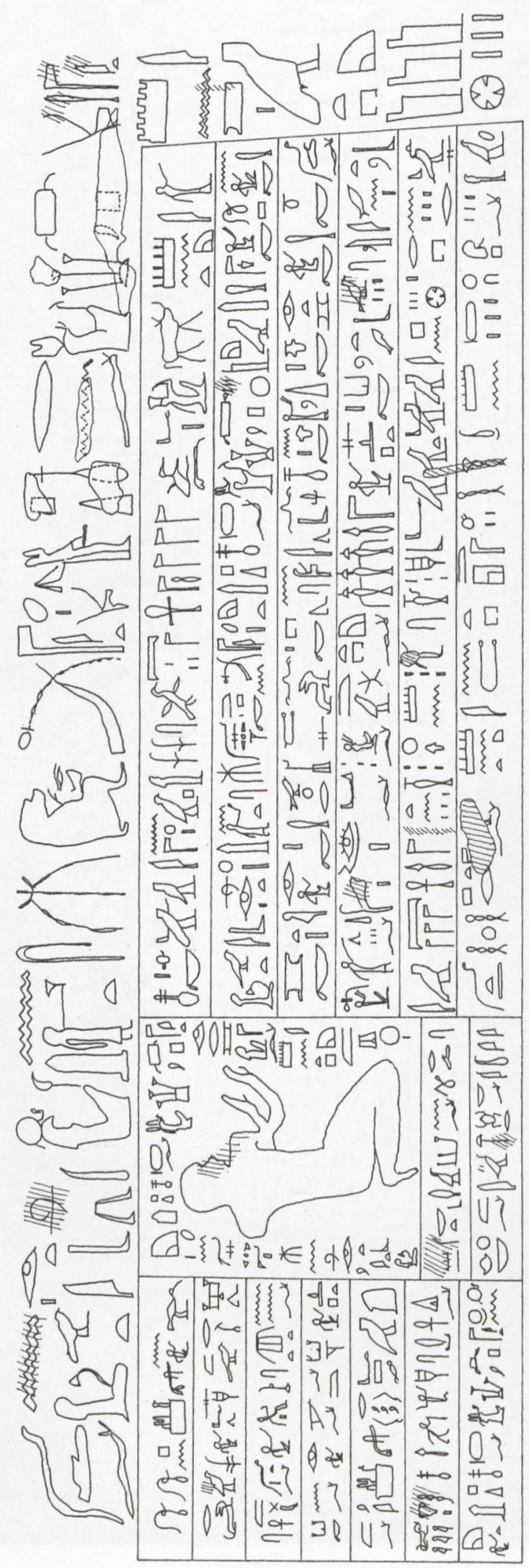

Inschrift des Amunpriesters K3p.f-ḩ3-Mntw , Luxortempel, Vorhof Amenophis' III., Zeichnung des Verfassers (zu Jansen-Winkeln, Ein Priester als Restaurator) 